\title{
Re-Dispatching Generation to Increase Power System Security Margin and Support Low Voltage Bus
}

\author{
Ronghai Wang, Student Member, IEEE, and Robert H. Lasseter, Fellow, IEEE
}

\begin{abstract}
This article proposes a new method to increase a power system's security margin and/or support its low voltage bus by re-dispatching generator outputs, using a normal vector found at a voltage collapse boundary or a low voltage boundary (LVB). This method uses the normal vector as an indicator to change the generation direction so that more power can be transferred before reaching a boundary of a critical limit such as the voltage collapse boundary or LVB, etc. The method has been tested in the simulation on several systems and shows predicted results. It can be applied to many practical power systems to enhance the system's security, or to increase the system's transfer capability. It also provides technical guidance for an open access transmission in a deregulated environment, when bidding results are shown as generation patterns.
\end{abstract}

Index Terms-power generation dispatch, power system control, power system dynamic stability, power system reliability, power system scheduling, power system security, power transmission control, power transmission reliability.

\section{INTRODUCTION}

$\mathbf{P}$ OWER system reliability problems are caused by many factors. The generation pattern and the load pattern, which represents generation and load at every bus, are among the leading factors. A poorly scheduled generation or load pattern can reduce a system's ability to transfer power while maintaining its reliability. Studies on the economic dispatch problem assume that the system can maintain its reliability. The optimal power flow (OPF) program does consider both the economic dispatch and the stability, but it requires a heavy computation. With the open access transmission in a deregulated environment, poorly scheduled generation patterns and load patterns from competitive bidding will be seen more and more often. These patterns might cause many stability problems.

Between the power system generation pattern and the load pattern, the generation pattern is easier to control. The load pattern is relatively uncontrollable due to the uncontrollable consumer demand. Although load-sheddings and price incentives can be used as ways to adjust the load pattern, these are not generally recommended except under extreme conditions such as at peak load or under contingencies. On the other hand, a generation pattern has more flexibility in terms of supplying power. It

Manuscript received August 5, 1998; revised July 1, 1999.

The authors are with the Department of Electrical and Computer Engineering, University of Wisconsin, Madison, WI 53706 USA (e-mail: ronghai @cae.wisc.edu).

Publisher Item Identifier S 0885-8950(00)03778-0. is easier to control than the load pattern by the utility companies or an independent system operator (ISO).

Another reason for considering the generation pattern is that the generation pattern can cause more problems if not controlled properly. Normally there are more load buses than generator buses in a common power system and these load buses are usually highly mesh (network) connected. They are more stable (in terms of voltage stability) than scattered generator buses. Industrial experiences have shown that even a remote generator shutdown can cause severe voltage instability.

A good generation direction (or pattern) should be maintained to supply the maximum power possible to the load before reaching the boundary of a system limit. The boundary of the limit can be a voltage collapse boundary (also called the Point of Collapse (PoC) boundary [2]), a low voltage boundary (LVB), or a thermal limit boundary, etc. To form a good generation direction, sometimes a generator needs to reduce its power output so that other generators can transfer more power to the load. That is why utilities under heavy peak load prefer local generation to buying cheaper power from far-away generators when the system reliability is critical. In the power industry, economy is always secondary to reliability. However, when local generation is insufficient, remote generation is still necessary. In a competitive utility market, buying power from far-away generators will be very common. The method proposed in this paper gives guidance to the cooperation among generators (power companies) on how to form a good generation direction to avoid technical difficulties in transmission.

Much work has been done in a load space to control the load direction [1], [2] to avoid the system limits, while few work have been done in the generation space. The methods developed in the load space might not be very useful in a generation space. The minimum distance to the PoC boundary in a load PQ space can be determined by the method proposed in Dobson's paper [3], [4]. In addition, the minimum distance to the PoC boundary in a generation space can be obtained in a similar way. However, the distance to the boundary of a limit is not the same as the total generation on the boundary of the limit. The above method cannot find the generation direction for the maximum possible total generation by simply changing the sign of the objective function. The minimum distance to the boundary of a limit is in the $L_{2}$ norm form (such as $|X|_{2} \equiv \sqrt{\sum x_{i}^{2}}$ ) in the load PQ space as in most of the papers [3], [4]. It might be good to use the $L_{2}$ norm form for security reasons such as in the case of finding the minimum distance to the PoC boundary. However, in a generation space the maximum total generation on the boundary of a limit is expressed better in the $L_{1}$ norm form (such 
as $\left.|X|_{1} \equiv \sum\left|x_{i}\right|\right)$, since the amount of power generation and its price are always calculated in the $L_{1}$ norm forms.

The method in this paper deals with the generation space. It finds the generation direction that maximizes the total generation on the boundary of a limit. A new gradient search algorithm is proposed to find this generation direction using a normal vector found at the boundary of the limit. The paper is organized as follows: Section II discusses the normal vector on the boundary surface. Section III introduces the search algorithm to maximize the generation capability. Simulation results are also presented. Section IV summarizes the method, its applications and future work.

\section{ThE NoRmal Vector at THE BOUndary}

\section{A. Traditional Powerflow Equation and Jacobian}

The traditional power flow equation $F=0$ can be written in a complex number form as:

$$
F \equiv V I^{*}-S=V(Y V)^{*}-S=0
$$

where

$$
V=\left[\begin{array}{c}
\left|V_{1}\right| e^{j \theta_{1}} \\
\left|V_{2}\right| e^{j \theta_{2}} \\
\vdots \\
\left|V_{n}\right| e^{j \theta_{n}}
\end{array}\right], S=\left[\begin{array}{c}
S_{1} \\
S_{2} \\
\vdots \\
S_{n}
\end{array}\right]=\left[\begin{array}{c}
P_{1} \\
P_{2} \\
\vdots \\
P_{n}
\end{array}\right]+j\left[\begin{array}{c}
Q_{1} \\
Q_{2} \\
\vdots \\
Q_{n}
\end{array}\right]
$$

Here $n$ is the number of total system buses, $V$ is the complex vector of the bus voltage including magnitudes and angles, $I=$ $Y V$ is the complex bus injection current vector, $Y$ is an $n \times n$ system complex admittance matrix, $S$ is the bus complex power injection vector, $j=\sqrt{-1}$ is the imaginary unit number, $P$ and $Q$ are the bus active and reactive power injection vectors, respectively.

The $n$ system buses are grouped into $m P V$ buses and $(n-m)$ $P Q$ buses. Generally, $P V$ buses are generator buses with known bus voltage magnitudes and generator active power outputs, except one $P V$ bus (usually bus 1), which is chosen as the slack generator bus. The slack bus has a known voltage magnitude and its active power output is slack so as to balance the system power loss. The slack bus is usually also chosen as the voltage angle reference bus with a zero phase angle. $P Q$ buses are load buses with known active power and reactive power injections. The power flow equation solves for a state variable vector $x$ to match all $(m-l) \mathrm{PV}$ bus active power outputs (except slack bus), all $(n-m)$ PQ bus active power and $(n-m)$ reactive power outputs.

The state variable $x$ includes all $(n-l)$ bus voltage angles (except the reference angle) and all $(n-m)$ PQ bus voltage magnitudes. It has the form of

$$
x=\left[\cdots, \theta_{r}, \cdots,\left|V_{s}\right|, \cdots\right]^{T}, \quad x \in R^{2 n-m-1}
$$

where $r$ is for all $(n-l)$ buses (except the angle reference bus), $s$ is for all $(n-m)$ PQ buses. The power flow Jacobian $F_{x}$ is a $(2 n-m-l) \times(2 n-m-1)$ matrix.

\section{B. Modified Powerflow Equation and Jacobian}

When the system load is increased along an assumed direction, the amount of generator active power outputs to balance the load increase need to be calculated. To do this, let $K_{P L}$ and $K_{Q L}$ denote the direction vectors of the system load active power and reactive power increase. Let $K_{P G}$ be the direction unit vector of the generator active power increase. $K_{P L}$ and $K_{P G}$ are all unit vectors in the $L_{1}$ norm forms. Then the bus complex power injection $S$ under the generation increase without including generator reactive power can be expressed as

$$
S=S_{0}+K_{P G} \times g-\left(K_{P L}-j K_{Q L}\right) \times l
$$

where

$S_{0} \quad$ is the base case,

$l \quad$ is a scalar that represents the amount of load increase and

$g$ is a scalar for corresponding generator outputs.

The above equation does not include the generator reactive power output pattern since the generator reactive power is not considered in the power flow equation, as a generator bus is a PV bus. The generator's reactive power will be determined after the power flow equation is solved. If a generator reactive power limit is reached, we will turn this generator from a PV bus into a PQ bus with a fixed reactive power output at the limit, and add that reactive power into (3). Now assume $g$ is a system parameter and is increased from zero to a predetermined value. To balance the modified power flow equation $F=0$, we need to solve for the state variables $x$ and the slack variable $l$ for the parameter $g$. Here all generators' active power outputs are fixed by $K_{P G} \times g$, and all load increase is along the direction of $\left(K_{P L}+j K_{Q L}\right)$. This modified power flow equation solves for the state variable $y$ to match all $m$ PV bus active power outputs, all $(n-m)$ PQ bus active power and $(n-m)$ reactive power outputs. The state variable $y$ has the form of

$$
y=\left[l, \cdots, \theta_{r}, \cdots,\left|V_{s}\right|, \cdots\right]^{T}=\left[l, x^{T}\right]^{T}, \quad y \in R^{2 n-m}
$$

where $r$ is for all $(n-l)$ buses (except the angle reference bus), $s$ is for all $(n-m)$ PQ buses. The modified power flow Jacobian $F_{y}$ is a $(2 n-m) \times(2 n-m)$ matrix.

It can be easily shown that the traditional power flow equation is only a special case of the modified power flow equation when the generation direction is on the previous slack generator and $g$ is used as the slack variable instead of $l$. The advantage of the modified power flow equation is that it will keep the generator direction and the load direction as fixed while increasing the generation and the load till reaching the PoC boundary. Unlike the traditional power flow equation, all generator buses and load buses are included in the modified power flow equation. The modified power flow equation contains information of the generation direction and the load direction. It is also convenient for calculating the normal vector in a full generation space since there is no singled-out slack generator bus, as to be discussed later. 


\section{The Normal Vector to the PoC Boundary Surface in a Generation Space}

To study the maximum possible total generation of a system, or a system's maximum transfer capability, $g$ should be chosen as the parameter. Similar to what has been done in load space [3], [4], it can be shown that as $g$ increases to its maximum $g *$, a saddle node bifurcation occurs. At this maximum point $(y *, g *)$, we have

$$
\begin{array}{r}
F(y *, g *)=0 \\
\left.F_{y}\right|_{*} \text { has reduced rank of }(2 n-m-1) \\
\left.F_{y}\right|_{*} \text { has a single zero eigenvalue } \\
\left.\omega F_{g}\right|_{*} \neq 0 \\
\left.\omega F_{y y}\right|_{*}(v, v) \neq 0
\end{array}
$$

where $\omega$ and $v$ are nonzero left and right eigenvectors satisfying $\left.\omega F_{y}\right|_{*}=0$ and $\left.F_{y}\right|_{*} v=0$.

Assuming the load direction $\left(K_{P L}+j K_{Q L}\right)$ is pre-specified, in a generation space, where the axes are individual generator outputs $P_{G}$, a PoC boundary forms one point for each generation direction $K_{P G}$. When $K_{P G}$ scans over all possible directions, a hypersurface is formed by the points. The power flow equation will have no solution outside the hypersurface.

On the hypersurface, we have

$$
\left.F_{y}\right|_{*} d y+\left.F_{P_{G}}\right|_{*} d P_{G}=0, \quad P_{G} \in R^{m}
$$

or

$$
\left.\omega F_{y}\right|_{*} d y+\left.\omega F_{P_{G}}\right|_{*} d P_{G}=0, \quad P_{G} \in R^{m} .
$$

Since $\left.\omega F_{y}\right|_{*}=0$, we have $\left.\omega F_{P_{G}}\right|_{*} d P_{G}=0$, which means that the vector, $u=\left(\left.\omega F_{\Gamma_{G}}\right|_{*}\right)^{T}, u \in R^{m}$, is the normal vector to any $d_{P_{G}}$ lying on the hypersurface.

\section{The Normal Vector to the LVB Surface in a Generation Space}

Practically, a power system might not be loaded to its PoC boundary. Before reaching the PoC boundary, some bus voltages might be so low that the voltage-sensitive circuit breakers will separate the buses from the rest of power system, even this may cause cascade circuit breaker actions. The low voltage threshold of the circuit breaker is set at a predetermined acceptable limit regardless whether the system is at its $\mathrm{PoC}$ boundary or not. Considering this kind of situation, the LVB can be defined. A system is loaded to its LVB when any one of its buses reaches its preset low voltage limit, under a fixed generator direction and a load direction. At any point on the hypersurface of the LVB formed by scanning all possible generation directions in the generation space, there is one bus with its voltage at its preset low voltage limit.

Taking this constraint into consideration, the power flow equation satisfying the LVB conditions can be written as:

$$
F(Z)=0
$$

where

$$
z=\left[l, g, \cdots, \theta_{r}, \cdots\left|V_{q}\right|, \cdots\right]^{T}, \quad z \in R^{2 n-m} .
$$

Here $r$ is for all $(n-l)$ buses (except the angle reference bus), $q$ is for all $(n-m-l)$ PQ buses (the voltage magnitude of the load bus at its low voltage limit is known here and is not part of the unknown state variables). The power flow Jacobian $F_{z}$ is a $(2 n-m) \times(2 n-m)$ matrix.

Similar to the method that has been used in the optimal economic dispatch [5], considering the variable $g$, the total slack power generation, as the cost function or the constraint function, then the normal vector to the hypersurface of the LVB can be calculated as

$$
u=\frac{d g}{d P_{G}}=\frac{d g}{d F} \times F_{P_{G}} \quad u \in R^{m}
$$

where $\frac{d g}{d F}$ is the second row of the new inverse jacobian $F_{z}^{-1}$ since $g$ happens to be the second element of $z$, and $F_{P_{G}}$ can be obtained in the same way as $F_{P_{G}}$ in (6), which is a matrix with only a few ones as the elements.

\section{ThE GRAdiENT SEARCH Method}

\section{A. The Search Algorithm}

Now let's consider how to change $K_{P G}$ to increase the total generation $g$ before reaching a boundary. The optimization problem is stated as:

$$
\begin{aligned}
& \text { Max: }\left|g \times K_{P G}\right|_{1} \\
& \text { subject to: }\left|K_{P G}\right|_{1} \equiv \sum K_{P G}=1 \\
& \text { PoC boundary condition } \\
&(\text { or LVB condition })
\end{aligned}
$$

To solve the problem, let's consider the following property in a space. For a continuous and smooth hypersurface in a multi-dimensional hyperspace (e.g., the PoC boundary hypersurface or the LVB hypersurface in the $m$ dimension $P_{G}$ hyperspace), assume that the surface is convex enough, at the local maximum of the $L_{1}$ norm $\left(\left.\left.\left|P_{G}\right|_{\max }\right|_{1} \equiv \sum P_{G}\right|_{\max }\right.$ at $\left.P_{G}\right|_{\max }$ for example), it can be easily shown that at this point the normal vector to the hypersurface has the form:

$$
s=u\left(\left.P_{G}\right|_{\max }\right)=\frac{1}{\sqrt{m}}[1,1,1, \cdots]^{T} \quad s \in R^{m}
$$

It also means that at the point $\left.P_{G}\right|_{\max }$, the hypersurface of the boundary intersects a hyperplane which is tangent to the hypersurface at the point $\left.P_{G}\right|_{\max }$. All the points on the hyperplane have the same $L_{1}$ norm value as the $L_{1}$ norm value of $\left.P_{G}\right|_{\max }$ (which is $\left.\sum P_{G}\right|_{\max }$ ).

To solve the optimization problem using the above space property, we can choose

$$
\Delta K_{P G}=-\frac{u-(u \bullet s) s}{|u-(u \bullet s) s|_{2}} h
$$

where $u$ is the normal vector of a point on the hypersurface and $h$ is the step size. The new generation direction is updated by

$$
K_{P G}^{n e w}=K_{P G}+\Delta K_{P G}
$$

After normalizing $\left|K_{P G}^{n e w}\right|_{1}=\sum K_{P G}^{\text {new }}=1$, we calculate the system boundary again and obtain new $g^{\text {new }}$ and $u^{\text {new }}$. 


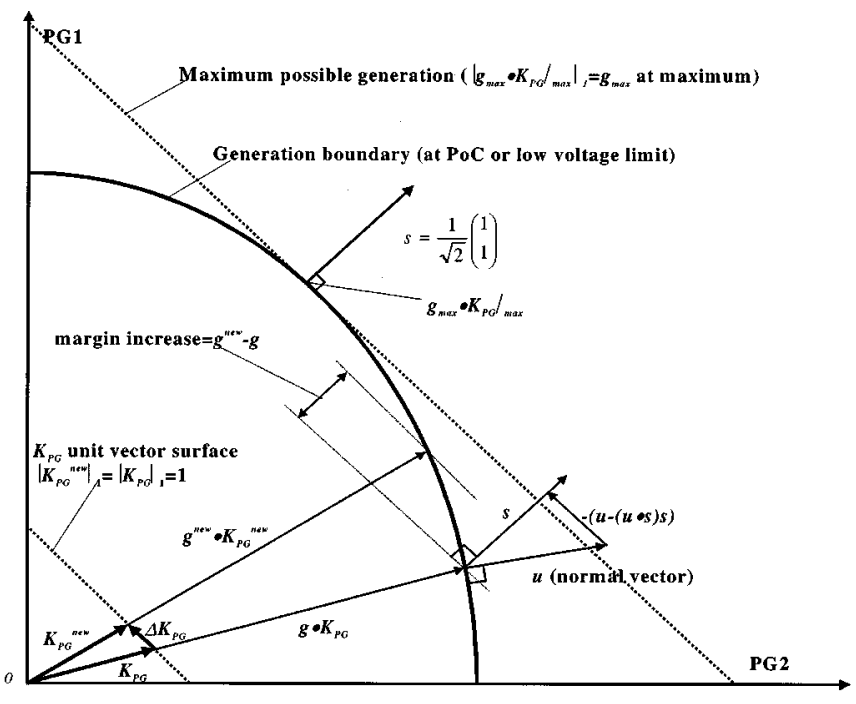

Fig. 1. The search algorithm.

This process repeats until the stop criterion is met. The ending $\left.K_{P G}\right|_{\max }$ is then the generation direction along which we can supply the maximum power to the system before the boundary is reached. At the boundary along $\left.K_{P G}\right|_{\max }$, the normal vector $u$ equals $s$.

Fig. 1 shows an example in a two generator system for a fixed load direction using this method. Since there are only two generators in the power system, the generation $P_{G}$ space is two-dimensional. The boundary of a limit can be either a PoC boundary or a low voltage boundary, and it is a curve here (hypersurface in a higher dimension hyperspace). At the boundary of the maximum possible total generation in the $L_{1}$ norm form, the boundary surface is tangent to a line (hyperplane in a higher dimension) with a normal direction $s=\frac{1}{\sqrt{2}}[1,1]^{T}$. Initially assuming we start from a generation direction $K_{P G}$ and get to the boundary at $g \times K_{P G}$, from the normal vector $u$ at this boundary, we can calculate the change for $K_{P G},-(u-(u \bullet s) s)$ to increase $g$. This vector of change is parallel to the line of the maximum possible total generation. By setting the step size $h$, we obtain the change in the generation direction, $\Delta K_{P G}$, from (13). The new generation direction $K_{P G}^{\text {new }}$ is updated as in (14). Since $\Delta K_{P G}$ stays on the line of the $K_{P G}$ unit vector, $K_{P G}^{\text {new }}$ will still have the $L_{1}$ norm of 1 . We then calculate the new boundary along $K_{P G}^{\text {new }}$ and get the new possible total generation $g^{\text {new }}$ on the boundary point of $g^{n e w} \times K_{P G}^{n e w}$. If the chosen step size $h$ is not too big, $g^{\text {new }}$ will be larger than $g$. This process will be repeated until $u=s$, or $g_{\max }$ along $\left.K_{P G}\right|_{\max }$ is reached. In this example we assume the base case is zero, which is not necessary.

Some points about the method need to be mentioned here. When calculating $g \times K_{P G}$ on the LVB, if the PoC boundary is reached before the LVB is reached, we will stop at the PoC boundary and use the normal vector on the PoC boundary instead.

The method can consider generator MW limits as well. If a generator reaches its MW limit, (or if we need to fix a generator's active power output at a specified value and consider only other generators), we can drop the generator from the generation

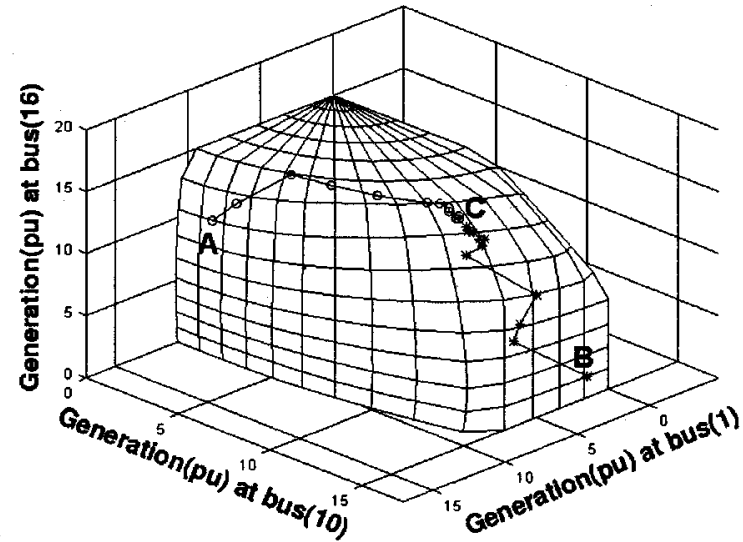

(a)

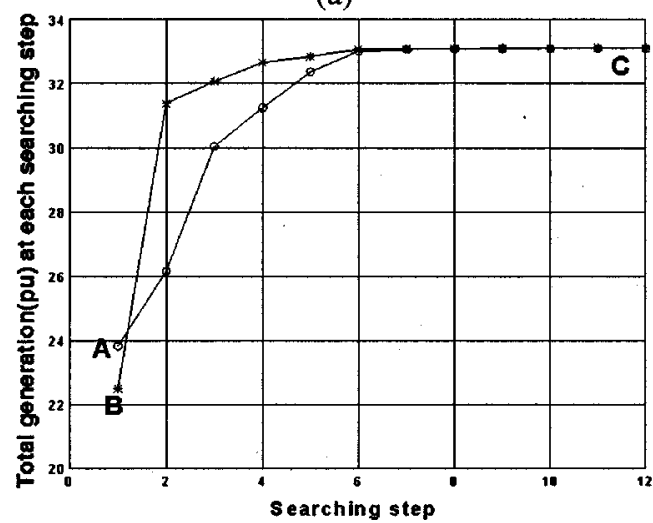

(b)

Fig. 2. Searching the $K_{P G}$ for the maximum generation capability: (a) On the surface, and (b) at each step.

space while keeping this generator's output at its MW limit (or the specified output) in the subsequent calculations. So the dimensionality of the generation space is reduced by 1 . The method remains unchanged in the reduced dimension.

\section{B. Simulation}

To test this method in simulation, we modified the IEEE 24 bus system [6] into an 18 bus system with only three generators at bus 1, 10 and 16, and one synchronous condenser, so the surface of the boundary of a limit in the generation space is a three-dimensional surface here and can be visualized. As shown in Fig. 2, first, the boundary surface is calculated and plotted. The load direction is fixed as pre-specified and is the same for all generation directions here. The surface shown is a $0.9 \mathrm{pu}$ LVB surface. To form the surface, for all possible generation directions, the generation parameter $g$ is increased using a continuation power flow program until any of the bus voltage drops to the low voltage limit, which is 0.9 pu for all 18 buses in this case, or stops at the $\mathrm{PoC}$ boundary, whichever is reached first. Thus all stopping boundary points form the surface. We further set all generator MW limits at 15 per unit (100 MVA base). So the surface is restricted in the bus 10 and the bus 16 directions.

To test the method, we choose to start at a boundary point A. It takes about 12 steps to get to the final boundary point C, which has the direction of the maximum total generation. 
TABLE I

GENERATOR OUTPUTS During SEARCH FROM POINT A to POINT C

\begin{tabular}{|c|c|c|c|c|c|c|c|}
\hline step & 1 & 2 & 3 & 4 & 5 & 6 & 12 \\
\hline Gen 1(pu) & 10.6 & 10.3 & 9.5 & 9.1 & 8.5 & 7.6 & 7.2 \\
\hline Gen 10(pu) & 2.6 & 3.6 & 5.5 & 7.1 & 8.8 & 10.4 & 11.6 \\
\hline Gen 16(pu) & 10.6 & 12.2 & 15.0 & 15.0 & 15.0 & 15.0 & 14.3 \\
\hline Total $(\mathrm{pu})$ & 23.8 & 26.2 & 30.0 & 31.2 & 32.4 & 33.0 & 33.1 \\
\hline
\end{tabular}

At point $\mathbf{C}$, its normal vector is, $u=s=\frac{1}{\sqrt{3}}[1,1,1]^{T}$. We also start at a different boundary point $\mathbf{B}$, which ends at point $\mathbf{C}$ as well. Fig. 2(a) shows every steps on the surface. Fig. 2(b) shows that at each step, the total generation at the boundary is increased. We need to keep the step size $h$ small enough so that at the next step the total generation at the boundary is increased. During the search, we also need to keep the step size $h$ large enough so we can reach the final point $\mathbf{C}$ in fewer steps. For this reason, a variable step size algorithm is implemented along with the search algorithm.

Table I shows the first few steps when the search algorithm change generation pattern from point A to point C. Notice step 1 is the point $\mathrm{A}$; step 12 is the point $\mathrm{C}$. It clearly shows that the total generation at the boundary increases as the generation pattern changes. Notice generator 16 reached its MW limit at 15 pu during the search and finally reduced. And generator 1 has to reduce its active power in order to transfer more total power.

We also tested the method using the original IEEE 24 bus system that has 10 generators, with MW, MVAR limits and low voltage limits. In the 10-dimension generation hyperspace, the method gives similar results with improved security margin or transfer capability.

The CPU time in our test ranges from 30 seconds to a few minutes on the HP C-180 unix station in MATLAB environment. It should be noticed that the time mainly depends on the type of the computer used, the size of the system, the initial generation direction started from, the precision requirements of the final results, and the other computational technique involved.

\section{DISCUSSION}

Besides the PoC boundary or LVB, the method can also be used to consider any other operational limits. With added constraint functions, boundaries can be found for system limits, such as line current limits. After the normal vector is found on those boundaries, the search algorithm can be performed to adjust the generation direction and avoid those limits as well.

Although the method can be used to consider many different system operational limits, it should be noted that for any limit this method is the fastest ascent search method. Of the many paths existing between the starting searching point and the final point with the maximum total generation, a system operator will have many choices to adjust generator dispatch before the limit for the maximum total generation is reached. A system operator might choose, for example, to do economic dispatch, with in mind the knowledge of how much more power can be safely generated and transmitted.

Although we have only tested this methods on simple load models so far, it can be shown that adding complicated voltagedependent load models will not change the flexibility of the method, only complicate the constraint equation and the power flow Jacobian. Future work can be done with different available load models.

This method assumes that the load direction is fixed. In the real world the load direction might change every minute. As the load direction changes, the search algorithm should be done again for the new load direction to find a new generation direction. With a reasonable load forecast model incorporated, using fast computing methods such as the parallel processing method or the sparse matrix method, on-line implementation of this method will be possible. This will greatly benefit an open access market when the validity of bidding results has to be verified (and possibly modified) under reliability constraints in a timely manner. The number of steps needed to get to the final generation direction depends on the initial generation direction and it will determine the speed of the search algorithm. To reduce the steps, after we get the final point, we can use it as the initial point once the load direction is changed slightly and we need to search again. In this way the computation time can be greatly reduced in on-line implementation. To further reduce the computation time, a direct method instead of the search algorithm can be developed in future work.

This method is able to find a local maximum only. If we start from the current generation direction, we should be able to get to the nearest local maximum. However, multiple maximums may coexist in a system since the surface of the boundary of a limit might not be smooth. To find those maximums, we could start the search from multiple different initial points to find them. However there is no guarantee that all of them can be found.

This method should be performed under the worst first contingency condition as well, because a power system often operates assuming this condition for reason of the reliability. For example, if we find a generation direction with the boundary maximized under a worst line contingency case and use it in normal operation, the system will be safe under any line outage conditions while maintaining the maximum power transfer capability. Further, the search can also be pre-performed for each contingency and put into operating procedures, so a system operator knows how to re-dispatch generation should a contingency occur.

\section{CONCLUSION}

This paper demonstrates a method of adjusting the generation direction to increase the power system transfer capability up to the system's voltage collapse boundary or LVB. The advantage of this method to any OPF program is obvious: the use of the normal vector eliminates the need to calculate the sensitivities of the individual generator output that may need more power flow iterations.

This method will be helpful in an open access transmission environment, where generators supply power according to the pricing strategy before any system stability limit is reached. When a system operates near a LVB or a PoC boundary, increasing reactive power generation sometimes can be used to enhance reliability. However, this might be in conflict with system voltage regulation requirements. If this is the case, the system operator should encourage different generators to form a certain 
generation direction using this method in order to transfer more power to the load.

\section{ACKNOWLEDGMENT}

The authors gratefully acknowledge the contribution of Ian Dobson and Scott Greene for helpful discussions.

\section{REFERENCES}

[1] S. Greene, I. Dobson, and F. L. Alvarado, "Sensitivity of the loading margin to voltage collapse with respect to arbitrary parameters," IEEE Transaction on Power Systems, vol. 12, no. 1, pp. 262-272, February 1997.

[2] C. A. Cañizares, "Voltage collapse and transient enagy function analysis of ac/dc systems," $\mathrm{PhD}$ dissertation, University of Wisconsin-Madison, 1991.

[3] I. Dobson and L. Lu, "New method for computing a closest saddle node bifurcation and worst case load power margin for voltage collapse," IEEE Transaction on Power Systems, vol. 8, no. 3, pp. 905-913, August 1993.

[4] I. Dobson, "Computing a closest bifurcation instability in multidimensional parameter space," Journal of Nonlinear Science, vol. 3, pp. 307-327, 1993.
[5] J. Arrillaga and C. P. Arnold, Computer Analysis of Power Systems: John Wiley \& Sons, 1990.

[6] "EKE reliability test system," IEEE Transactions on Power Apparatus and Systems, vol. PAS-98, no. 6, pp. 2047-2054, Nov./Dec. 1979.

Ronghai Wang (S'92) received the B.S. degree from Nanjing Univ., P.R. China in 1989, and an M.S. from Michigan Technological Univ. in 1992, both in physics. He is currently a Ph.D. student in the Electrical and Computer Engineering Department of University of Wisconsin-Madison. His main interest is in power electronics and powa system analysis, including power transfer capability and FACTS devices.

Robert H. Lasseter (F'92) received the Ph.D. degree in physics from the University of Pennsylvania, Philadelphia, in 1971. He was a Consultant Engineer with the General Electric Company until 1980 when he joined the University of Wisconsin-Madison. His main interest is the application of power electronics to utility systems, including hardware, methods of analysis and simulation. 